several European Countries, and can be considered as a prevention strategy in Europe

\title{
HIV-screening in pregnant women: a systematic review of cost-effectiveness studies
}

\section{Fabrizio Bert}

F Bert ${ }^{1}$, MR Gualano ${ }^{1}, P$ Biancone ${ }^{2}, V$ Brescia $^{2}$, E Camussi $^{1}$, $M$ Martorana $^{1}$, S Secinaro ${ }^{2}, R$ Siliquini $^{1}$

${ }^{1}$ Department of Public Health Sciences, University of Turin, Turin, Italy ${ }^{2}$ Department of Management, University of Turin, Turin, Italy Contact: fabrizio.bert@unito.it

\section{Background}

Vertical transmission represents the major route of HIV infection for children; however, the preventive interventions available are extremely effective. Then, we aim to summarize the existing evidences regarding the cost-effectiveness of mother-to-child-transmission preventive screenings, in order to help policy makers in choosing the optimal antenatal screening strategy.

Methods

We conducted a systematic review of studies investigating the cost-effectiveness of HIV-screening addressed to pregnant women following PRISMA guidelines, using three databases: PubMed, Scopus and Cost-Effectiveness Analysis Registry (CEA).

\section{Results}

The final selection obtained 21 papers. Part of the studies assessed the cost-effectiveness of antenatal HIV-screening during early gestation. Others estimated the cost-effectiveness of HIV-screening in late pregnancy. The selected papers focused on both developed and developing countries, characterized by different HIV prevalence. The characteristics and methodology of the retrieved studies were heterogeneous. However, all studies agreed on main findings, outlining the cost-effectiveness of HIV-screening. Cost-effectiveness ratio improves increasing HIV burden. The major findings proved robust across the various scenarios tested in sensitivity analysis. Discussion

Our review confirmed the cost-effectiveness not only of HIV antenatal screening, but also of rescreening in late gestation in both developed and developing countries. In fact, universal screening resulted cost-effective even with extremely low HIV prevalence. One of the most influencing parameter was women acceptance rate. Therefore, maximize screening coverage appears a priority worldwide. Further studies assessing the optimal HIV-test choice and the best testing sequence for confirmation, could be useful and of primary importance for the European public health agenda.

\section{Key messages:}

- Our review confirmed the cost-effectiveness not only of HIV antenatal screening, but also of rescreening in late gestation in both developed and developing countries

- HIV-screening for pregnant women resulted cost-effective even when the HIV prevalence is extremely low, common in 